\title{
Atomically Dispersed Precious Metal Species on Various Oxide Supports for Catalytic Hydrogen Upgrading and Emission Control
}

\author{
Ming Yang, ${ }^{1,2}$ Lawrence F. Allard ${ }^{3}$ and Maria Flytzani-Stephanopoulos ${ }^{1}$ \\ 1. Department of Chemical and Biological Engineering, Tufts University, Medford, MA 02155, USA \\ 2. Chemical and Materials Systems Laboratory, General Motors Global Research and Development, \\ Warren, MI 48090, USA \\ 3. Materials Science \&Technology Div., Oak Ridge National Laboratory, Oak Ridge, TN 37831, USA
}

Sir Hugh Stott Taylor first defined the concept of "catalytic active sites" in 1925 as the place where the catalytic reaction occurs. In a homogeneous phase, the active sites are uniquely defined, but on a heterogeneous catalyst surface, it is always challenging to identify the active sites; the latter often change with the operating conditions and are disguised by the presence of nanoparticles (NPs), which typically contain many metal atoms that do not catalyze the reactions [1]. Prior work at Tufts has established that sub-nm Au and Pt species (undetectable by regular TEM) catalyze the low-temperature water-gas shift (WGS) reaction [2, 3]. The concept of single-site heterogeneous catalysts may bring together homogeneous and heterogeneous catalysis. In the heterogeneous catalysts systems, how to best identify the exact active sites at the atomic scale, and to maximize the exact structures so that they remain stable under the reaction conditions is challenging. In the present work, it is shown that atomically dispersed single-site Pt and Au species can be prepared on various support oxides to meet diverse fuel gas processing reaction scenarios (Figure 1). Aberration-corrected high-angle annular darkfield STEM imaging is particularly useful for characterizing single-atom catalyst dispersions.

Starting from the heavily investigated $\mathrm{TiO}_{2}$-supported $\mathrm{Au}$ nanocatalysts, this work describes appreciable loadings $(\sim 1 \mathrm{wt} . \%)$ of isolated $\mathrm{Au}$ atoms bound on titania and shows that single-atom gold with vicinal -OH species catalyzes the low-temperature WGS reaction [4]. The catalyst preparation combines a typical Au deposition-precipitation method with UV irradiation, followed by the removal of the weakly bound Au NPs by cyanide leaching. This work provides new evidence that atomically dispersed $\mathrm{Au}-\mathrm{O}_{\mathrm{x}}-$ $(\mathrm{OH})_{\mathrm{y}}$ species bound on the titania surface are the active sites for the WGS reaction, the same as on other reducible oxide supports (e.g. ceria and iron oxide). An important question is whether such singlesite $\mathrm{Au}$ species can be prepared stably on any support oxide, including inert oxide substrates (e.g. zeolites and silica), preferably through facile preparation techniques.

This exploration began with Pt [3], where our group at Tufts had previously discovered that alkali ions were necessary to disperse and activate Pt on alumina and silica supports, but identifying the Pt ion state was difficult in the presence of other structures of Pt. Also, direct transfer of this knowledge to Au originally failed. We have refined the preparation conditions and showed that single-Pt-atom centric clusters are formed through $\mathrm{Pt}-\mathrm{O}_{\mathrm{x}}-\mathrm{Na}$ linkages, the ensembles being equally effective on supports as diverse as $\mathrm{TiO}_{2}$, KLTL zeolites, and mesoporous silica MCM-41 [5]. Loading of $0.5 \mathrm{wt} \%$ Pt on all these supports preserves the $\mathrm{Pt}$ in atomic dispersion as $\mathrm{Pt}(\mathrm{II})-\mathrm{O}_{\mathrm{x}}-\mathrm{Na}($ or $\mathrm{K})(\mathrm{OH})_{\mathrm{y}}$ - species catalyzing the WGS reaction from below 150 to $400{ }^{\circ} \mathrm{C}$. The same preparation protocol was next applied to $\mathrm{Au}$, a better choice than Pt for the low-temperature WGS due to its lower apparent activation energy for this reaction $(\sim 45 \mathrm{~kJ} / \mathrm{mol}$ for $\mathrm{Au}$ vs. $\sim 70 \mathrm{~kJ} / \mathrm{mol}$ for Pt) [6]. The intrinsic activity of the single-site $\mathrm{Au}$ species is similar on inert supports (KLTL zeolites and mesoporous silica MCM-41) as on ceria, iron oxide, and titania supports; apparently all sharing a common, similarly structured Au active site. Based on these 
findings, a one-step "green" route was explored to create exclusively single-gold-atom centric clusters stabilized by $-\mathrm{O}-\mathrm{Na}$ linkages. The new $\mathrm{Au}-\mathrm{TiO}_{2}$ catalysts show stable high activity for both the WGS and methanol steam reforming reactions. For the first time, a simple green impregnation route is shown to produce a highly active and stable $\mathrm{Au}$ catalyst, and this may be adopted for the industrial preparation/application of Au catalysts, as direct as those used for the Pt group metal (PGM) catalysts.

General principles for catalyst design at the single-atom limit have emerged from this work to be applied to other catalytic reaction systems beyond the hydrogen-producing reactions, specifically for costeffective and durable catalytic systems for ultra-low emission control in the next-generation gasoline and diesel vehicles. Our new findings at GM R\&D unambiguously confirm the advantages of the atomically dispersed PGM catalysts in improving $\mathrm{CO}$ and $\mathrm{HC}$ conversions and de $\mathrm{NO}_{\mathrm{x}}$ selectivity, even after severe hydrothermal ageing reflecting the typical vehicle lifecycle of 150k miles. [7]

\section{References:}

[1] J.M. Thomas, Nature 2015, 525, 325.

[2] Q. Fu et al., Science 2003, 301, 935.

[3] Y. Zhai et al., Science 2010, 329, 1633.

[4] M. Yang et al., J. Am. Chem. Soc. 2013, 135, 3768.

[5] M. Yang et al., J. Am. Chem. Soc. 2015, 137, 3470.

[6] M. Yang et al., Science 2014, 346, 1498.

[7] Microscopy research at ORNL sponsored in part by the U.S. Dept. of Energy (DOE), Office of Energy Efficiency and Renewable Energy, Vehicle Technologies Program, as part of the Propulsion Materials Program, and by DOE Basic Energy Science under Grant \# DE-FG02-05ER15730 to Tufts University.

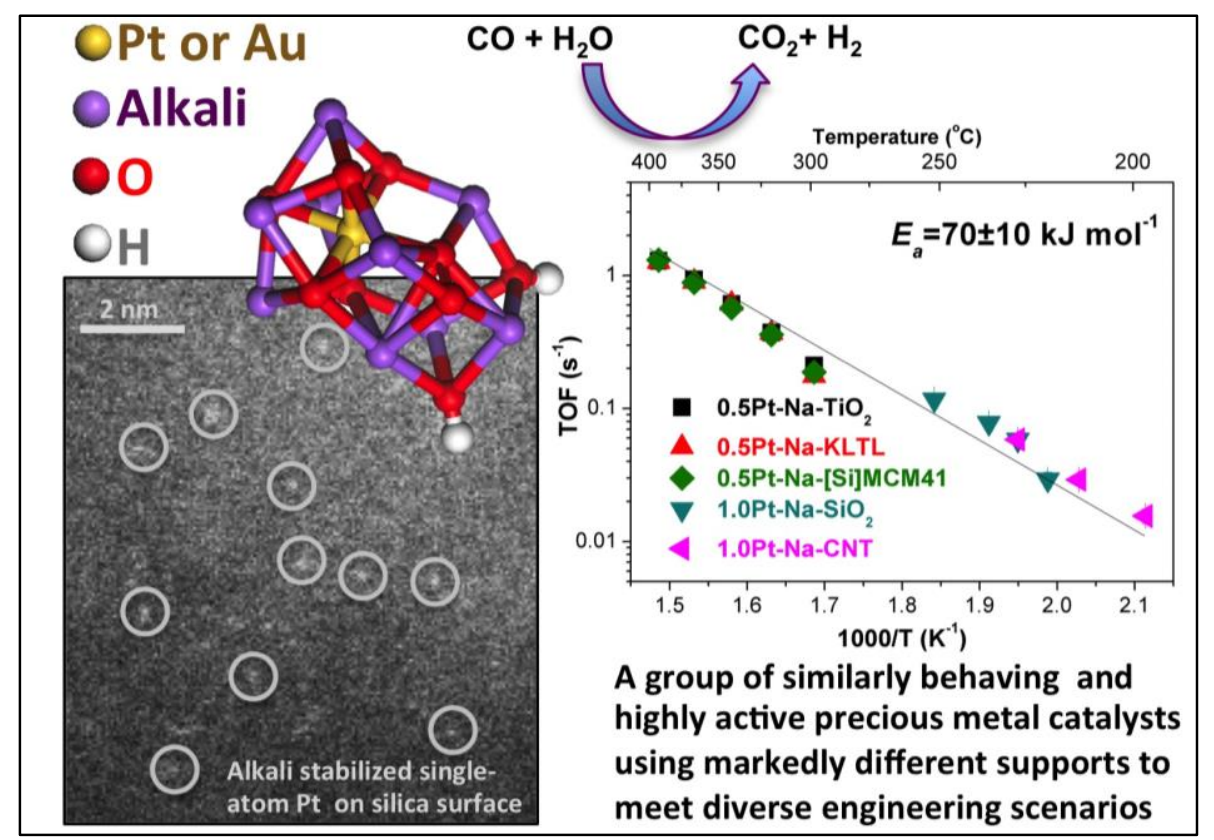

Figure 1. Atomically dispersed $\mathrm{Pt}$ and $\mathrm{Au}$ species have been stably anchored on various supports to meet diverse application scenarios. The common single-atom-centric $\mathrm{Pt}$ (or $\mathrm{Au}$ ) $-\mathrm{O}_{\mathrm{x}}-\mathrm{Na}(\mathrm{OH})_{\mathrm{y}^{-}}$species has been confirmed with various experimental tools, and the HAADF-STEM imaging has been key to the identification. These exclusive single-sites catalyze the low-temperature water-gas shift reaction with equal atomic efficiency, and the support effects are indirect. 